disease than untreated mice. Deposition of IgG and complement 3 was significantly less in the TwHF groups, although levels of autoantibodies to double-stranded DNA increased during treatment for all three groups. Splenomegaly and normalization of splenic architecture occurred only in TwHF-treated mice.

The authors conclude that an ethyl acetate extract of TwHF could provide a novel therapeutic approach in SLE patients.

Rachel Murphy

Original article Tao X et al. (2006) Therapeutic impact of the ethyl acetate extract of Tripterygium wilfordii Hook F on nephritis in NZB/W F1 mice. Arth Res Ther 8: R24

\section{Experience with bosentan in pulmonary arterial hypertension secondary to systemic sclerosis}

The FDA approved bosentan for treatment of pulmonary arterial hypertension $(\mathrm{PAH})$ in patients with systemic sclerosis in December 2001. Trials that reported efficacy for bosentan in PAH included heterogeneous patient populations, however, and the few treated patients with PAH secondary to systemic sclerosis did not improve their exercise capacity. Joglekar et al., therefore, aimed to broaden the clinical experience with bosentan in patients with this rare condition. They retrospectively assessed the efficacy and tolerability of bosentan by comparing their results with those of previously reported studies, because a placebo arm would have been unethical.

The 23 patients were given $62.5 \mathrm{mg}$ bosentan twice daily for the first month, followed by $125 \mathrm{mg}$ twice daily for the remaining 17 months. One patient was lost to follow-up after 6 months and three permanently discontinued bosentan after 9 months, because of increased transaminase levels (two patients) or fluid retention (one patient), perhaps indicating a higher risk of liver toxicity for patients with PAH secondary to systemic sclerosis, compared to those with $\mathrm{PAH}$ of other causes.

The patients showed improvements in their overall function scores during the first 3 months of treatment, followed by stabilization between 3 and 6 months, and a trend towards declining function after 12 months. The authors state that the improvement in overall function scores was at least as good as in previously reported trials. No improvement was seen in pulmonary functioning, however, from which they conclude that bosentan does not influence the restrictive lung disease of these patients. As with many studies, however, more questions are raised than answered.

\section{Caroline Barranco}

Original article Joglekar A et al. (2006) Bosentan in pulmonary arterial hypertension secondary to scleroderma. J Rheumatol 33: 61-68

\section{B-cell depletion as a treatment for refractory SLE}

Two recent, uncontrolled studies have shown that B-cell depletion with rituximab, and repeated B-cell depletion with rituximab, are effective in treating systemic lupus erythematosus (SLE) patients with active disease refractory to conventional immunosuppressive therapy.

In the first study, 24 patients received varying regimens of rituximab, cyclophosphamide and steroids; all but one patient achieved B-cell depletion lasting 3-8 months, and one of these remained depleted at 4 years. Serum complement 3 levels, anti-double-stranded DNA antibody levels and global British Isles Lupus Assessment Group scores improved from the time of B-cell treatment to 6 months post-treatment.

In the second trial, seven patients who were successfully treated in the first trial received additional cycles of treatment after relapse of disease; in total, four patients had three cycles of treatment and three patients had two cycles. The mean time to re-treatment was 13 months. After the second treatment cycle, four of the seven patients had improved British Isles Lupus Assessment Group scores with corresponding improvements in anti-double-stranded DNA antibody and complement 3 levels. After the third treatment cycle, two of the four patients improved. Re-treatment was generally well tolerated.

The authors conclude that B-cell depletion is an attractive option in patients with refractory SLE. Randomized, double-blind, controlled trials of this treatment are warranted.

Rachel Murphy

Original articles Leandro MJ et al. (2005) B-cell depletion in the treatment of patients with systemic lupus erythematosus: a longitudinal analysis of 24 patients. Rheumatology 44 : 1542-1545

$\mathrm{Ng} \mathrm{KP}$ et al. (2005) Repeated B cell depletion in treatment of refractory systemic lupus erythematosus. Ann Rheum Dis [doi:10.1136/ard.2005.044487] 